\title{
Thoracic Endovascular Aortic Repair in Patients with Prior Open Aortic Surgery
}

\author{
Hideyuki Shimizu, MD, PhD, Akihiro Yoshitake, MD, PhD, Satoshi Kawaguchi, MD, \\ Shinji Kawaguchi, MD, Hidenobu Takaki, MD, and Ryohei Yozu, MD, PhD
}

\begin{abstract}
Objective: To review our experience of thoracic endovascular aortic repair (TEVAR) in patients with prior open aortic repair (OAR).

Materials and Methods: Stent-grafts were deployed in the arch, descending thoracic and thoracoabdominal aortae of 39, 13 and 5 patients, respectively, and in a deteriorated extra-anatomical prosthesis in one. The access route was the femoral artery in 10 of 23 patients with, and in 30 of 35 patients without a prior abdominal prosthesis. Prior prostheses and elephant trunks comprised 57 of 116 landing zones and 23 proximal landing zones, respectively.

Results: Three patients died before discharge. Type II endoleaks developed in six patients, and Types I and III developed in one patient each. Type I endoleaks were not found at landing zones comprising prosthetic grafts. The overall actuarial three-year survival rate including early mortality was $86.5 \%$.

Conclusion: The clinical outcomes of TEVAR were excellent, even in patients with prior OAR. Prosthetic grafts, including elephant trunks, provided good landing zones for TEVAR. Prostheses with largercaliber designs are recommended for iliac artery reconstruction in future TEVAR.
\end{abstract}

Keywords: aneurysm, reoperation, stent graft

\section{INTRODUCTION}

Tndications for thoracic endovascular aortic repair 1 (TEVAR) are rapidly expanding worldwide and patients who have undergone prior aortic surgeries have become candidates for this procedure. Some of them had new or recurrent aneurysms late after primary surgery, and others had extensive aortic pathology that was treated as part of a two-stage hybrid procedure comprising open aortic repair (OAR) and subsequent TEVAR. The present study reviews our experience with TEVAR in patients with prior OAR.

Department of Cardiovascular Surgery, Keio University, Tokyo, Japan

Received: September 13, 2012; Accepted: January 24, 2013 Corresponding author: Hideyuki Shimizu, MD, PhD. Department of Cardiovascular Surgery, Keio University, 35 Shinanomachi, Shinjuku-ku, Tokyo 160-8582, Japan

Tel: +81-3-3353-1211, Fax: +81-3-5379-3034

E-mail: shimizu.md@gmail.com

\section{Materials ANd Methods}

Fifty-eight patients (male, $\mathrm{n}=48$; female, $\mathrm{n}=10$; mean age, 75.3 years; range, $48-89$ years) with a history of OAR underwent TEVAR between January 2001 and May 2012. The number of prior aortic surgeries was 1, 2, 3 and 4 in 38, 15, 3 and 2 patients, respectively, and the sites of surgery were the ascending, arch, the descending, thoracoabdominal and abdominal aortae in 5, 27, 24, 6 and 23 patients, respectively. Aortic pathology comprised atherosclerotic aneurysm $(n=45)$, aortic dissection $(\mathrm{n}=5)$, pseudoaneurysm $(\mathrm{n}=6)$ and dilation of a deteriorated prosthetic graft $\left.(n=2) .^{1,2}\right)$ Aneurysms treated by TEVAR were located in the arch, the descending thoracic aorta, and the thoracoabdominal aorta in 39, 13,5 patients, respectively and in an extra-anatomical prosthetic graft in one patient. Nine and 5 patients with aneurysm in the aortic arch and in the thoracoabdominal aorta, respectively, underwent a single-stage hybrid procedure, in which major branch arteries were 
debranched, bypasses to these vessels were created, and stent-grafts were positioned.

The sites of stent-graft insertion were the femoral artery (via inguinal incision), abdominal aorta/iliac artery (via laparotomy), descending aorta (via left thoracotomy) and the ascending aorta (via median sternotomy) $)^{3)}$ in $40,14,1$ and 3 patients, respectively. The femoral artery was the access route in $10(43.5 \%)$ of 23 patients with a history of a replaced abdominal aorta and/or iliac artery. On the other hand, the femoral artery was used as an access route in $30(85.7 \%)$ of 35 patients who did not have prior surgery for abdominal aorta and/or iliac artery. The difference was statistically significant $(\mathrm{p}=0.001)$.

The indications for laparotomy in 14 patients were to secure an appropriate access route when the femoral approach was impossible ( $\mathrm{n}=8$ patients) due to a previously implanted prosthesis with a small caliber or abnormal flexion, concomitant open repair of a juxtarenal abdominal aortic aneurysm that was unsuitable for endovascular repair $(n=1$ patient) and hybrid repair of thoracoabdominal aortic aneurysms with debranching and rerouting visceral and renal arteries ( $n=5$ patients). A 10-mm diameter prosthetic graft was implanted and used as an access route for TEVAR in nine patients. The tug-of-wire technique, in which a guide wire is passed from the right brachial to the femoral artery, was applied in 22 patients.

The stent-grafts used for TEVAR consisted of GORE TAG $(\mathrm{n}=22$ patients), Talent $(\mathrm{n}=14)$, Zenith TX2 $(\mathrm{n}=8)$, Valiant $(\mathrm{n}=3)$, GORE TAG + Talent $(\mathrm{n}=2)$, branched Inoue $(n=2)$, fenestrated Najuta $(n=2)$ and others $(n=5)$. The size of the stent-graft was determined based on pre-procedural measurements using computed tomography $(\mathrm{CT})$. The measured diameter of a prior prosthesis was usually a few millimeters larger than its nominal diameter at implantation.

Fifty-seven of 116 landing zones consisted of prior prostheses; 40 were proximal and 17 were distal. A prosthetic graft was located at only the proximal landing zone, only the distal landing zone, at both and none in 27, 4, 13 and 14 patients, respectively. Among them, 23 underwent TEVAR after total arch replacement with an elephant trunk as a part of scheduled staged hybrid repair for extensive arch aneurysm. Thus, elephant trunks served as proximal landing zones in these patients.

The interval between the first open aortic surgery and TEVAR ranged from 27 days to 40 years (mean, 9.4 years; median, 7.5 years).

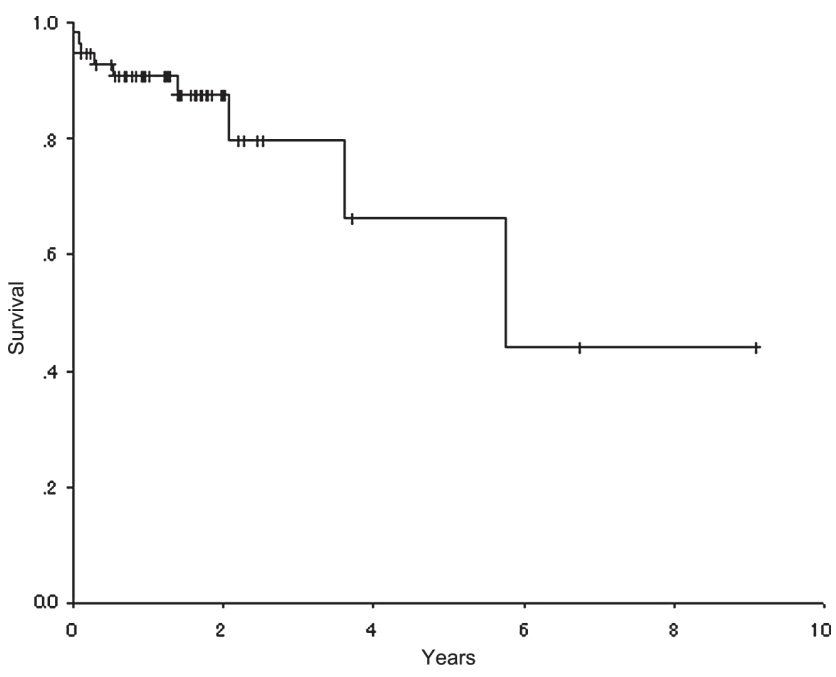

Fig. 1 Actuarial survival rates including early mortality.

\section{Results}

\section{Operative mortality}

Three patients died due to rupture of thoracoabdominal aortic aneurysms $(n=1)$, worsening interstitial pneumonia $(\mathrm{n}=1)$ and cerebral infarction $(\mathrm{n}=1)$ before discharge (hospital mortality rate, 5.2\%). All three patients who died had required laparotomy for hybrid repair of a thoracoabdominal aortic aneurysm $(n=2)$ and to establish an access route for TEVAR because the previous Y-shaped prosthesis was too small $(n=1)$. Postoperative major morbidity included cerebral infarction $(\mathrm{n}=2)$, delayed paraparesis $(\mathrm{n}=1)$, respiratory failure requiring tracheotomy $(n=2)$ and hemodialysis $(n=1)$.

\section{Postoperative evaluation by computed tomography}

Except for two patients who died soon after intervention, 56 patients underwent enhanced postoperative CT. Type II endoleaks were detected in six patients, and Types I and III developed in one patient each. Type I endoleaks were not found at landing zones consisting of prosthetic grafts. No mortality or endoleaks occurred among those who underwent two-stage hybrid arch repair or TEVAR with an elephant trunk for the proximal landing.

\section{Actuarial survival rates and rates of freedom from aorta-related mortality}

Actuarial survival rates at 1, 3, 5 and 7 years were $90.9 \%$ (SD, 3.9\%), 79.7\% (SD, 8.8\%), 66.4\% (SD, 14.2\%) and $44.3 \%$ (SD, 20.4\%), respectively (Fig. 1), and rates of 


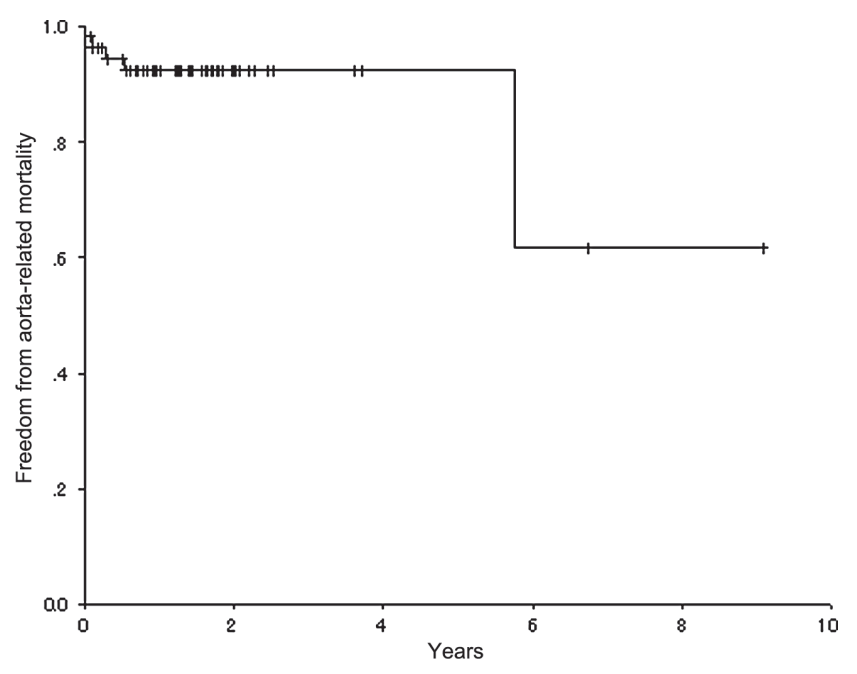

Fig. 2 Rates of freedom from aorta-related mortality.

freedom from aorta-related mortality were $92.5 \%$ (SD, $3.6 \%$ ), $92.5 \%$ (SD, 3.6\%), $92.5 \%$ (SD, 3.6\%) and $61.7 \%$ (SD, 25.3\%), respectively (Fig. 2).

\section{Discussion}

Repeated aortic surgery is required for failed prior surgeries such as pseudoaneurysm formation at anastomosis sites, dilation of prostheses, new or recurrent aortic lesions after primary aortic surgery, and for extensive aortic pathology as part of elective staged repair to avoid invasive single-stage repair.

Generally, repeated open surgery is technically challenging and associated with high mortality and morbidity. Etz and colleagues identified a $13.3 \%$, hospital mortality rate for repeated descending and thoracoabdominal aortic repair and found that respiratory complications arose in $23.6 \%$ of patients. $\left.{ }^{4}\right)$ They also described that reoperations often take longer and even recommend a two-day procedure for patients with extensive adhesions. To avoid such cumbersome procedures and minimize the invasiveness of reoperations, TEVAR is undoubtedly a useful alternative.

Several hybrid aortic reconstructive techniques using thoracic endograft technology combined with some surgical techniques have recently been developed in an attempt to improve surgical outcomes, especially in patients who were previously considered as being at prohibitively high risk for conventional open repair of aortic aneurysms. ${ }^{5)}$ Two-stage hybrid repair can treat extensive thoracic aortic aneurysms. However, the choice of singleor two-stage repair remains controversial. Kouchoukos and colleagues reported good results from single-stage repair, with a hospital mortality rate of $6.5 \%$. However, the relatively high morbidity rate remains a concern as it is often accompanied by reoperation for bleeding (17\%), mechanical ventilation $>72$ hours $(42 \%)$, temporary tracheotomy (13\%) and temporal renal dialysis (9\%). ${ }^{6)}$ On the other hand, Safi, et al. ${ }^{7)}$ and Etz, et al. ${ }^{8}$ reported that staged repair of extensive aortic aneurysms, or a combination of the first-stage proximal reconstruction using the open arch elephant trunk technique and a secondstage operation through a left thoracotomy, improves neurological outcomes. However, the entire repair cannot be completed in some patients due to either complications during first-stage repair or the magnitude of the second-stage operation, which is a concern. Two-stage hybrid repair, or the elephant trunk repair with complete endovascular repair, can avoid a morbid incision and shorten the interval to the second stage. $\left.{ }^{9}\right) \mathrm{Kim}$ and colleagues $^{10)}$ reported that more patients complete the second-stage of two-stage hybrid repairs and that the second-stage hospital stay is shorter and fewer transfusions are required compared with elephant trunk repair and surgical completion. The outcomes of two-stage hybrid repair for extensive aortic arch aneurysms in our series were also excellent. Even in patients with a shaggy aorta, no cerebral complications developed after twostage hybrid repair, possibly because the diseased arch aorta was already replaced with a prosthesis at the time of TEVAR, which seems useful for preventing postoperative stroke.

However, room for improvement remains in hybrid repair of thoracoabdominal aortic aneurysms consisting of visceral artery debranching and revascularization followed by TEVAR. Our results were consistent with those of Chiesa and colleagues, who described a high perioperative mortality of $23 \%$ and a morbidity (renal failure, respiratory failure and delayed transient paraplegia) rate of $30.8 \% .{ }^{11)}$ Such high mortality and morbidity rates might be attributable to the need for laparotomy during hybrid repair of thoracoabdominal aortic aneurysms to revascularize visceral and renal arteries. A far less invasive procedure, such as total endovascular repair using a branched stent-graft or a fenestrated stent-graft, is required.

An indication for TEVAR, accurate measurement of landing zones and proper device selection are key factors in preventing type I endoleaks. Although instructions for use note that stent-graft systems have not been evaluated in patients who have undergone previous surgical repair in the thoracic and/or abdominal aortic area, prior 
prostheses seem to serve as good landing zones based on our experience. The size of a stent-graft was determined in our series based on pre-implant CT measurements and the measured diameter of the previous prosthesis was a few millimeters larger than its nominal diameter at implantation. Takami and colleagues reported that the diameter of a graft implanted in the thoracic aorta increases by $26 \%$ compared with the package size immediately after implantation, and becomes further dilated by about $10 \%$, compared with the diameter at discharge, at a rate of $3.23 \%$ per year for up to five years after surgery. ${ }^{12)}$ Thus, the device size should be based, not on the nominal diameter of the prior prosthesis, but on the actual diameter when the prosthesis is used as a landing zone.

A visionary strategy during the initial open surgery should be considered for future TEVAR at least when the adjacent part of a replaced aorta is diseased and might require future treatment. For example, a prosthesis with a larger-caliber design for the iliac artery could provide an adequate access route. A prosthesis with a diameter similar to the opposite landing site of a future stent-graft could prevent a significant size discrepancy between proximal and the distal landing zones. Adequate distance should be maintained between the major branch and a possible landing zone to prevent occlusion of the major branch by a possible stent-graft. Better-prepared strategies would surely help to further improve outcomes.

\section{Conclusions}

The outcomes of endovascular aortic repair for thoracic aorta pathology among patients with a history of OAR are satisfactory. Prosthetic grafts provide good landing zones for endovascular repair and the value of two-stage hybrid repair is confirmed. When undergoing surgery to treat an abdominal aorta/iliac artery, the use of prostheses with larger-caliber designs is recommended, especially when future endovascular repair is a possibility.

\section{Disclosure Statement}

None.

\section{ReFERENCES}

1) Anzai T, Shimizu H, Yozu R, et al. Repeated transluminal endovascular Inoue stent graft placement for progressive dilatation of prosthetic graft that had been repaired with Inoue stent graft placement for dilatation 23 years after extraanatomical bypass. Gen Thorac Cardiovasc Surg 2007; 55: 76-9.

2) Shimizu H, Hashimoto S, Kuribayashi S, et al. Images in cardio-thoracic surgery: Dilation of a prosthetic aortic graft 23 years after implantation. Eur J Cardiothorac Surg 2009; 35: 906.

3) Shimizu H, Hachiya T, Yamabe K, et al. Hybrid arch repair including supra-aortic debranching on the descending aorta. Ann Thorac Surg 2011; 92: 2266-8.

4) Etz CD, Zoli S, Kari FA, et al. Redo lateral thoracotomy for reoperative descending and thoracoabdominal aortic repair: a consecutive series of 60 patients. Ann Thorac Surg 2009; 88: 758-66; discussion 767.

5) Szeto WY, Bavaria JE. Hybrid repair of aortic arch aneurysms: combined open arch reconstruction and endovascular repair. Semin Thorac Cardiovasc Surg 2009; 21: 347-54.

6) Kouchoukos NT, Mauney MC, Masetti P, et al. Singlestage repair of extensive thoracic aortic aneurysms: experience with the arch-first technique and bilateral anterior thoracotomy. J Thorac Cardiovasc Surg 2004; 128: $669-76$.

7) Safi HJ, Miller CC, Iliopoulos DC, et al. Staged repair of extensive aortic aneurysm: improved neurologic outcome. Ann Surg 1997; 226: 599-605.

8) Etz CD, Zoli S, Mueller CS, et al. Staged repair significantly reduces paraplegia rate after extensive thoracoabdominal aortic aneurysm repair. J Thorac Cardiovasc Surg 2010; 139: 1464-72.

9) Obitsu Y, Koizumi N, Iida Y, et al. Long-term results of second-stage thoracic endovascular aortic repair following total aortic arch replacement. Gen Thorac Cardiovasc Surg 2010; 58: 501-5.

10) Kim T, Martin TD, Lee WA, et al. Evolution in the management of the total thoracic aorta. J Thorac Cardiovasc Surg 2009; 137: 627-34.

11) Chiesa R, Tshomba Y, Melissano G, et al. Hybrid approach to thoracoabdominal aortic aneurysms in patients with prior aortic surgery. J Vasc Surg 2007; 45: 1128-35.

12) Takami Y, Tajima $K$, Kato W, et al. Long-term size follow-up of knitted Dacron grafts (Gelseal ${ }^{\mathrm{TM}}$ ) used in the ascending aorta. Interact Cardiovasc Thorac Surg 2012; 14: 529-31. 